\title{
Actividad física para mejorar fuerza y equilibrio en el adulto mayor
}

\author{
Physical activity to improve strength and balance in old people
}

Luz Marina Chalapud-Narváez ${ }^{*}$ orcid.org/0000-0003-4047-7105

Armando Escobar-Almario' ${ }^{1}$ orcid.org/0000-0001-7731-6503

1 Facultad de Educación, Corporación Universitaria Autónoma del Cauca. Popayán, Colombia

\author{
Fecha de recepción: Septiembre 21 - $2016 \quad$ Fecha de revisión: Enero 17 - $2017 \quad$ Fecha de aceptación: Marzo 24 - 2017
}

Chalapud-Narváez LM, Escobar-Almario A. Actividad física para mejorar fuerza y equilibrio en el adulto mayor. Univ. Salud. 2017;19(1):94-101. DOI: http://dx.doi.org/10.22267/rus.171901.73

\begin{abstract}
Resumen
Introducción: La tercera edad abarca una época bastante amplia de la vida de un ser humano y la actividad física es una estrategia que permite mejorar la calidad de vida de esta población. Objetivo: Determinar la efectividad de un programa de actividad física, para mejorar la fuerza de miembros inferiores y el equilibrio en las personas de la tercera edad. Materiales y métodos: Estudio cuasi-experimental, de corte longitudinal, con una muestra de 57 personas de la tercera edad, a quienes se les aplicó una evaluación inicial y final de equilibrio y fuerza, y un programa de actividad física de 4 meses de duración, con dos sesiones de entrenamiento por semana, donde se realizaron ejercicios de postura, propiocepción, equilibrio y fuerza muscular. En el análisis de datos se utilizó el programa SPSS v. 24.0 para Windows. Se realizó análisis univariado aplicando medidas de tendencia central (media, mediana, moda, desviación estándar y rangos) y un análisis estadístico con pruebas no paramétricas para dos muestras relacionadas Wilcoxon. Resultados: Las siguientes pruebas arrojaron significancia estadística: extensión funcional $(\mathrm{p}=0,000)$, tándem ojos abiertos $(\mathrm{p}=0,20)$, tándem ojos cerrados $(\mathrm{p}=0,002)$, unipodal ojos cerrados $(\mathrm{p}=0,012) \mathrm{y}$ sentado/parado $(\mathrm{p}=0,000)$. Conclusión: Este estudio constató que la actividad física es efectiva para mejorar el equilibrio y la fuerza muscular de miembros inferiores y es una herramienta adecuada para conservar la funcionalidad y la autonomía de las personas de la tercera edad.
\end{abstract}

Palabras clave: Actividad física; adulto mayor; equilibrio postural; fuerza muscular. (Fuente: DeCS, Bireme).

\begin{abstract}
Introduction: Old age encompasses a fairly broad age in a person's life, and physical activity becomes the strategy to improve the elderly's life quality. Objective: To determine the effectiveness of a physical activity program in improving strength to the lower limbs and balance for the elderly. Materials and methods: Semi-experimental longitudinal study with a sample of 57 elderly people to whom an initial and final assessment of balance and strength was performed, as well as a 4 -week physical activity program with 2 training sessions each, comprising posture, proprioception, balance and muscle strength exercises. The program used for data analysis was SPSS v.24.0 for Windows. An univariate analysis was performed applying central trend measurements (mean, median, mode, standard deviation, range) and a statistical analysis with non-parametric testing for two related Wilcoxon samples. Results: The following tests showed statistical significance: Functional extension $(p=0,000)$, open eyes tandem $(p=0,20)$, closed eyes tandem $(p=0,002)$, closed eyes unipodal $(p=0,012)$ and sitting/standing $(p=0,000)$. Conclusion: This study determined that physical activity is effective to improve balance and muscle strength for lower limbs and a suitable tool to preserve senior's functionality and Independence.
\end{abstract}

Keywords: Motor/Physical activity; aged; balance/postural balance; muscle strength. (Source: DeCS, Bireme). 


\section{Introducción}

La tercera edad abarca una época bastante amplia de la vida de un ser humano, se puede afirmar que esta etapa inicia a partir de los 60 años ${ }^{1}$. El proceso de envejecimiento depende de la determinación genética, de los hábitos y del estilo de vida de cada individuo, siendo estos factores determinantes en la importancia de la diferenciación de la edad biológica y la edad cronológica con relación a la calidad de vida2,3. El ser humano evoluciona según la interacción de los factores biológicos y de los socioambiéntales, es decir, lo adquirido en el transcurso de la vida acelera el envejecimiento y lo convierte en un problema de salud pública ${ }^{4-6}$.

Ahora bien, en Colombia se evidenció que el porcentaje de población mayor de 60 años en el 2012 fue del 9,2\% de la población general, la esperanza de vida después de los 60 años es de 24 años, de los cuales 16,5 años son de vida saludable7 ${ }^{7}$ La pirámide poblacional se está invirtiendo, la perspectiva de envejecimiento a nivel mundial establece que la población de la tercera edad para el año 2030 equivaldrá al 16\% de la población mundial y que en el 2050 equivaldrá al $22 \%$, esto indica que las personas vivirán más y que por eso hay que buscar programas que ayuden a mejorar su calidad de vida $^{7-9}$.

Así pues, desde que inicia el proceso de envejecimiento, empieza a disminuir la funcionalidad del principal sistema del organismo humano, el sistema nervioso central8,10, suceso que desencadena una pérdida progresiva de las capacidades y habilidades relacionadas a todas las actividades del individuo en la vida diaria, entre ellas, una de las que más afecta a la población de la tercera edad es la deambulación, que necesita de fuerza, equilibrio, coordinación y autonomía ${ }^{8,11-15}$.

En consecuencia, se evidencia que el envejecimiento tiene una relación directa con el desarrollo de la discapacidad, hecho que está asociado a la disminución del movimiento y a deficiencias en la visión y la audición; lo anterior se puede relacionar a las caídas, accidentes frecuentes entre las personas de la tercera edad, dada la progresiva inestabilidad a edades avanzadas ${ }^{8}$. Entre los sujetos de edad superior a los 65 años, cerca del 23\% de los hombres y del $52 \%$ de las mujeres, han sufrido al menos una caída ${ }^{16}$, el número de caídas junto con la gravedad de las complicaciones es mayor cuando más edad tiene el sujeto17-19. Las caídas representan un factor de riesgo para la salud del adulto mayor, ya que, si no se previenen o se cuidan pueden generar una limitación funcional ${ }^{3}$.

Al menos un $60 \%$ de la población mundial no realiza la actividad física necesaria para obtener beneficios para la salud, por ello las personas de la tercera edad son una población en alto riesgo ${ }^{8,20}$. Según la Organización Mundial de la Salud (OMS), se recomienda que el adulto mayor realice "150 minutos de actividad física a la semana con una intensidad moderada-vigorosa para adulto mayor o 300 minutos de actividad leve"21. Teniendo en cuenta lo anterior, los programas de actividad física deben proponer ejercicios orientados a mejorar el equilibrio y la fuerza muscular de miembros inferiores, capacidades que permiten que el adulto mayor sea más estable en la deambulación previniendo las caídas y la aparición de factores asociados a la discapacidad 8,16,22.

Por tal razón, se planteó este estudio cuyo objetivo es determinar la efectividad de un programa de actividad física para mejorar el equilibrio y la fuerza muscular de los miembros inferiores de las personas de la tercera edad.

\section{Materiales y métodos}

El estudio cuasi-experimental es de tipo longitudinal, en el cual se contrastaron las siguientes hipótesis: La actividad física mejora la fuerza de miembros inferiores y el equilibrio del adulto mayor. Hipótesis nula: La actividad física no mejora la fuerza de miembros inferiores y el equilibrio del adulto mayor.

\section{Población y muestra}

La población estuvo conformada por grupos de la tercera edad de la comuna 2 de Popayán, la 
muestra se escogió a conveniencia y correspondió a 57 personas. Como criterio de inclusión se tuvo en cuenta que los participantes tuvieran 60 años o más y que pertenecieran a la comuna 2 de Popayán; fueron excluidas del estudio aquellas personas que no se encontraban presentes para la evaluación inicial, personas con inasistencia mayor del $20 \%$ al programa y sujetos que no aceptaron participar en el presente estudio.

\section{Instrumento}

Para el instrumento de recolección de información se retomaron las siguientes pruebas: 1) Extensión funcional o alcance funcional ${ }^{23-25 ;}$ 2) Prueba de Tándem²6; 3) Prueba unipodal o monopodal 17,24,27,28; 4) Prueba de sentado parado o Sit stand up ${ }^{29,30}$ validados por otros estudios, y que permitieron evaluar el equilibrio y fuerza muscular.

El test de extensión funcional mide la distancia que el participante puede alcanzar con su brazo extendido mientras permanece de pie sin desplazar sus pies $^{23}$. Se solicita al adulto mayor que se mantenga parado, con los pies juntos durante 60 segundos, con los ojos abiertos y cerrados $^{26}$. La prueba Unipodal mide el tiempo que se mantiene el participante sobre un pie, se realiza con ojos abiertos y cerrados ${ }^{27,28}$ y en la prueba sentado parado consiste en levantarse y sentarse de una silla el mayor número de veces ${ }^{29}$.

\section{Protocolo}

El programa de actividad física se realizó dos veces por semana con sesiones grupales, cada sesión tuvo una duración de 120 minutos con una intensidad de los ejercicios entre el 54\% al $75 \%$ de la frecuencia cardíaca máxima teórica.

En la primera semana se realizó la evaluación de los participantes. En la segunda y tercera semana se inició con ejercicios de posturas correctas en posición bípeda y sedente, ejercicios de reforzamiento muscular, ejercicios de equilibrio, con una intensidad moderada. En la cuarta y quinta semana se continuó con ejercicios de propiocepción, ejercicios de reforzamiento muscular, ejercicios de mejoramiento del equilibrio y estiramientos. En la sexta a onceava semana se realizaron ejercicios de reforzamiento muscular, ejercicios de mejoramiento del equilibrio y estiramientos. En cada semana se incrementó la intensidad y complejidad de los ejercicios. Para finalizar en la doceava semana se realizó la evaluación final y retroalimentación del programa.

\section{Procedimiento y análisis de la información}

La recolección de la información e implementación del programa de actividad física se realizó entre los meses de agosto a diciembre de 2014. La recopilación de información se llevó a cabo en las reuniones del grupo de tercera edad de la comuna 2, los días martes y jueves durante dos horas en la tarde. La población diligenció el consentimiento informado y prosiguió a brindar información general y a realizar la evaluación del equilibrio y fuerza muscular de miembros inferiores.

Para el análisis de los datos de la investigación, se utilizó el programa SPSS v.24.0 para Windows (SPSS Inc., Chicago, EE.UU.) con el fin de caracterizar sociodemográficamente a la población mediante la aplicación de encuestas y test de evaluación. De esta manera, se pudo identificar las condiciones de equilibrio y fuerza de miembros inferiores antes y después de aplicar el programa. Se realizó análisis univariado aplicando medidas de tendencia central, media, mediana, moda, desviación estándar y rangos, distribución de frecuencias. Además, para comparar los resultados de la evaluación del equilibrio antes y después de aplicar el programa, se realizó un análisis estadístico con pruebas no paramétricas para dos muestras relacionadas Wilcoxon, teniendo como referencia un nivel de significancia estadística valor de $\mathrm{p}<0,05$.

\section{Consideraciones éticas}

El estudio fue aprobado por el Comité de Ética del Sistema de Investigación de la Corporación Universitaria Autónoma del Cauca, quienes revisaron que los procedimientos de la investigación siguieran las consideraciones éticas de la declaración de Helsinki y la Resolución 8430 de 1993, clasificando el estudio en riesgo mínimo. También, se hizo diligenciar al 
participante un consentimiento informado, en el cual se le informa de los riesgos y beneficios de la investigación y de la confidencialidad de la identidad.

\section{Resultados}

La muestra de estudio estuvo conformada por 57 individuos con una edad promedio de 69 años aproximadamente con una desviación estándar (DS) de 9,3 años; el $26,3 \%$ de la población se encuentra entre los rangos de edad de 60 a 64 años. La mayoría de las personas son amas de casa y más de la mitad de la población pertenece al estrato socioeconómico 1 (Tabla 1).

El riesgo de caídas de la evaluación inicial y final, de las diferentes pruebas de evaluación aplicadas, presentó diferencias numéricas que permiten demostrar que hay cambios positivos en el equilibrio y fuerza muscular de miembros inferiores después de aplicado el programa de actividad física (Tabla 2).
Tabla 1. Características sociodemográficas de la población

\begin{tabular}{lrr}
\hline Característica & $\mathbf{n = 6 2}$ & (\%) \\
\hline Rango Edad & 15 & 26,3 \\
$60 \quad$ A 64 años & 11 & 19,3 \\
65 a 69 años & 13 & 22,8 \\
70 a 74 años & 8 & 14 \\
$75 \quad 79$ años & 10 & 17,5 \\
$>80$ años & & \\
Género & 50 & 87,7 \\
Femenino & 7 & 12,3 \\
Masculino & & \\
Ocupación & 47 & 82,5 \\
Ama de casa & 2 & 3,5 \\
Reciclador & 4 & 7 \\
Varios & 2 & 3,5 \\
Agricultor & 1 & 1,8 \\
Vendedor & 1 & 1,8 \\
Desempleado & & \\
Estrato & 32 & 43,9 \\
socioeconómico & & \\
1 bajo, bajo & 25 & \\
2 bajo & &
\end{tabular}

Tabla 2. Distribución de frecuencias de la evaluación inicial y final de las pruebas de equilibrio y fuerza de miembros inferiores de la población, según riesgo de caída y fuerza muscular de miembros inferiores

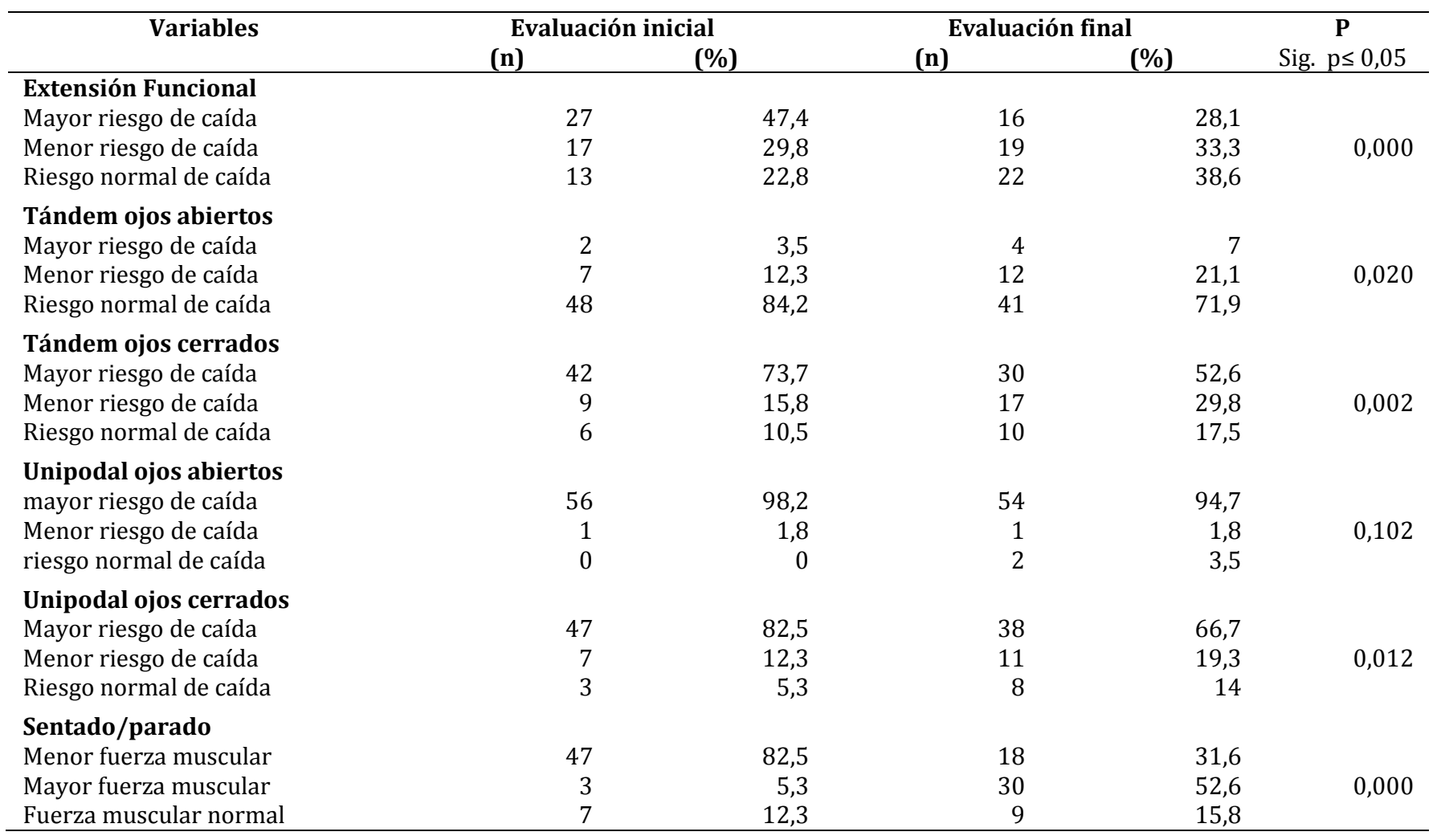


Se rechaza la hipótesis nula porque el nivel de significancia estadística es $\mathrm{p} \leq 0,05$ para las pruebas extensión funcional, tándem ojos abiertos y cerrados, unipodal ojos cerrados y sentado/parado, por lo tanto hay diferencias estadísticas en el nivel de riesgo de caídas de la población adulta mayor después de implementar el programa de actividad física (Tabla 3).

Tabla 3. Pruebas no paramétricas para dos muestras relacionadas (Wilcoxon) para determinar grado de significancia estadística de las evaluaciones iniciales y finales

\begin{tabular}{lcc}
\hline \multicolumn{1}{c}{ Característica } & \multicolumn{1}{c}{$\mathbf{P}$} & $\mathbf{Z}$ \\
\hline $\begin{array}{l}\text { extensión funcional final - } \\
\text { extensión funcional inicial }\end{array}$ & 0,05 & $-4,264$ \\
$\begin{array}{l}\text { tándem ojos abiertos final - } \\
\text { tándem ojos abiertos inicial }\end{array}$ & 0,020 & $-2,324$ \\
$\begin{array}{l}\text { tándem ojos cerrados final - } \\
\text { tándem ojos cerrados inicial }\end{array}$ & 0,002 & $-3,112$ \\
$\begin{array}{l}\text { unipodal ojos abiertos final - } \\
\text { unipodal ojos abiertos inicial }\end{array}$ & 0,102 & $-1,633$ \\
$\begin{array}{l}\text { unipodal ojos cerrados final - } \\
\text { unipodal ojos cerrados inicial } \\
\begin{array}{l}\text { sentado parado final - sentado } \\
\text { parado inicial }\end{array}\end{array}$ & 0,012 & $-2,500$ \\
\hline
\end{tabular}

\section{Discusión}

Esta investigación propuso un programa de tres meses de duración con dos sesiones semanales, aumentado la intensidad y complejidad de los ejercicios, con el fin de obtener beneficios perceptibles en la salud de los participantes. Esta propuesta se asemeja a estudios relacionados con el ejercicio físico, donde proponen trabajar sobre intensidades de entrenamiento moderadas y vigorosas, con una frecuencia de 2 a 5 días por semana, pero cuyo objetivo es modificar positivamente los factores de riesgo cardiovascular ${ }^{31}$. Además, teniendo en cuenta el aumento de la esperanza de vida, la promoción de la actividad física es indispensable para mejorar los procesos sociales de las personas de la tercera edad, ya que, como lo afirman Bhamani et al., "los problemas asociados con el estado funcional disminuido y la disminución de la actividad física en el avance de los años de vida se ha convertido en relevante" 32 .

Las pruebas que presentaron significancia estadística fueron extensión funcional, tándem ojos abiertos y cerrados, unipodal ojos cerrados y sentado parado, los resultados de unipodal ojos abiertos sólo presentaron cambios numéricos positivos. De igual forma, los hallazgos descritos en éste trabajo son similares con los resultados de estudios realizados sobre estos parámetros en países como Canadá y Francia ${ }^{23}$, y se han logrado efectos más notables comparado con estudios que cuentan con esquemas de entrenamiento afines $^{26,28,29}$.

Los programas de actividad física adaptados a las personas de la tercera edad que combinan ejercicios para mejorar la fuerza muscular de miembros inferiores, el equilibrio y que previenen las caídas, también son instrumentos apropiados para conservar la funcionalidad y la autonomía de las personas de la tercera edad.

En un estudio realizado se constató que las personas mayores de 65 años tienen más susceptibilidad de sufrir riesgo de caídas, debido a que la tasa de fallecimiento por estas aumenta de forma exponencial con el incremento de la edad, en ambos sexos y en todos los grupos raciales(33). Entre el $80 \%$ al $100 \%$ de la población de tercera edad de la comuna 2 de Popayán se encuentra en un rango de edad superior a 65 años, indicando que estas personas están en un riesgo mayor de sufrir un evento de caída. Con la implementación del programa y con los resultados obtenidos se puede pensar que se podría reducir esta tasa de fallecimiento.

Es de resaltar que el tiempo de duración del programa es de tres meses, siendo menor al utilizado en otros programas. Este estudio mostró significancia estadística mientras que estudios similares, cuyos tiempos de duración de ocho y nueve meses 28,29 obtuvieron resultados positivos, pero al realizar el análisis estadístico los cambios en estos, no mostraron significancia.

Los resultados positivos obtenidos en cuanto al progreso del equilibrio permitieron constatar la 
efectividad del programa en la población objeto, así como se ha reportado en estudios con programas afines ${ }^{23}$, lo cual se evidencia con la significancia estadística arrojada. Además, se observa que es importante mantener o mejorar el equilibrio en las personas de la tercera edad, ya que es una condición necesaria para realizar desplazamientos de una manera eficiente, hecho que a su vez disminuye el riesgo de caídas ${ }^{34}$.

La fuerza muscular de los miembros inferiores de la totalidad de los participantes en este estudio aumentó, presentando significancia en el análisis estadístico; evento que no sucedió en otra revisión donde se evaluó esta característica $^{30}$. Este aspecto no es referido ni discutido en otros estudios $26,28,29$. Algunos investigadores concluyen que la práctica regular de actividad física en el adulto mayor trae beneficios en su aptitud física, sobre todo en lo relacionado con la fuerza en miembros inferiores ${ }^{35}$.

La frecuencia de entrenamiento semanal que maneja el programa integral de equilibrio dinámico, al igual que el de otras revisiones ${ }^{28,29}$, está acorde con las últimas recomendaciones dadas por la OMS en cuanto al tiempo de actividad física recomendados para las personas mayores ${ }^{36}$.

En el programa las pruebas realizadas para evaluar el equilibrio ${ }^{37}$, no incluyen ninguna que lo valore dinámicamente, parámetro importante que se trabajó en esta investigación. Sería pertinente que también se implementen pruebas que midan de forma específica la parte dinámica de esta condición como lo hacen en otras revisiones ${ }^{22,38}$. Es de aclarar que entre las actividades contenidas en el programa se realizan ejercicios que refuerzan el equilibrio dinámico, aunque este no haya sido evaluado.

\section{Conclusiones}

Los resultados de este estudio constataron que la actividad física es efectiva para mejorar el equilibrio y la fuerza muscular de miembros inferiores, también es un instrumento adecuado para conservar la funcionalidad y la autonomía de las personas de la tercera edad.

De acuerdo con los resultados alcanzados en este estudio se sugiere generar alianzas de cooperación para los profesionales de la salud y la actividad física interesados en este tema, con el fin de proporcionar estrategias que permitan trabajar por el mejoramiento del equilibrio y la fuerza muscular de miembros inferiores en las personas de la tercera edad por medio de la actividad física.

Para la implementación de programas de actividad física se debe considerar las características socio-demográficas y culturales propias de la población para una mejor adaptación de los ejercicios con relación al contexto.

\section{Agradecimientos}

A la Corporación Universitaria Autónoma del Cauca, por brindar la oportunidad de realizar esta investigación y fomentar la cultura investigativa en su personal docente y cuerpo estudiantil.

Conflicto de intereses: Los autores no reportamos conflicto de intereses de ninguna clase.

\section{Referencias}

1. Lustosa LP, Silva JP, Coelho FM, Pereira DS, Parentoni AN, Pereira LSM. Impact of resistance exercise program on functional capacity and muscular strength of knee extensor in pre-frail community-dwelling older women: a randomized crossover trial. Rev Bras Fisioter [Internet]. 2011;15(4):318-24. Disponible en: http://www.ncbi.nlm.nih.gov/pubmed/21971726

2. Roig Hechavarría C, Ávila Fernández E, Mac Donal Bron D, Ávila Fernández B. La atención al Adulto mayor: un reto para la medicina contemporánea. Rev Cuba Med Gen Integr. 2015;31(3):346-52.

3. Cruz Quijano PD, Pérez Portal A, Piloto Rodríguez A, Díaz López D, Morales Izaguirre A, Reyes Herrera Y. Algunas causales relacionadas con caídas en el hogar del adulto mayor. Rev Cuba Med Gen Integr. 2014;311(1):35-41.

4. Pancorbo A. Medicina y ciencias del deporte y la actividad física. Vol. 5, Oceano. 2012.

5. Chong A. Aspectos biopsicosociales que inciden en la salud del adulto mayor. Rev Cuba Med Gen Integr. 2012;28(2):79-86.

6. Cavalli AS, Pogorzelski L de V, Domingues MR, Afonso 
M da R, Ribeiro JAB, Cavalli MO. Motivação de pessoas idosas para a prática de atividade física: estudo comparativo entre dois programas universitários Brasil e Portugal. Rev Bras Geriatr e Gerontol. 2014;17(2):255-64.

7. Organitation HelpAge International. Colombia, Latin America [Internet]. 2016. Disponible en: www.helpage.org/where-we-work/latinamerica/colombia/

8. Quintar E, Giber F. LAS CAÍDAS EN EL ADULTO MAYOR: FACTORES DE RIESGO Y CONSECUENCIAS. Actual osteol. 2014;10(3):278-86.

9. Botero de Mejía BE, Pico Merchán ME. Calidad de vida relacionada con la salud (CVRS) en adultos mayores de 60 años: una aproximación teórica. Hacia la Promoción la Salud. 2007;12(1):11-24.

10. Muir SW, Gopaul K, Montero Odasso MM. The role of cognitive impairment in fall risk among older adults: A systematic review and meta-analysis. Age Ageing. 2012;41(3):299-308.

11. Macedo D de O, Freitas LM de, Scheicher ME. Handgrip and functional mobility in elderly with different levels of physical activity. Fisioter e Pesqui. 2014;21(2):1515.

12. Salazar Pachón JD, Ramírez-Villada JF, Chaparro D. Revisión Sistemática Sobre el Impacto de la Actividad Física en los Trastornos de la Marcha en el Adulto Mayor. Apunt Educ Física y Deport. 2014;118(4):30-9.

13. Álvarez L, Ibarra L, García E. Eficacia de un programa para la promoción del bienestar a partir de las dimensiones del esquema de si mismo en un grupo de adultos mayores de la ciudad de Bucaramanga. Psychol Av la Discip. 2011;5(1):73-9.

14. Cerquera Córdoba AM, Flórez Jaimes LO, Linares Restrepo MM. Autopercepción de la salud en el adulto. Rev Virtual Univ Católica del Norte. 2010;31:407-28.

15. Cortes Muñoz C, Cardona Arango D, Segura Cardona AM, Garzòn Duque MO. Factores físicos y mentales asociados con la capacidad funcional del adulto mayor, Departamento de Antioquia, 2012. Rev Salud Pública. 2016;18(2):165-78.

16. Claros JAV, Cruz MVQ, Beltrán YH. Efectos del ejercicio físico en la condición física funcional y la estabilidad en adultos mayores. Rev Hacia la Promoción la Salud. 2012;17(2):79-90.

17. Pompei P, Murphy J. Gait Impairment and falls. geriartrics review syllabus; A core curriculum in geriatric medicine. Vol. 6, American Geriatrics Society. 2006. 195-209 p.

18. Tinetti M, Speechley M, Ginter S. Risk factors for falls among elderly persons living in the community. $\mathrm{N}$ Engl J Med. 1988;319(26):1701-7.

19. Galván Parra Y, Moreno Castillo Y, González Pedraza Avilés A. El síndrome de caídas y la calidad de vida relacionada con la salud en el adulto mayor. Arch en Med Fam. 2010;12(1):17-24.

20. Vidarte JA, Vélez C, Sandoval C, Alfonso ML. Actividad Física: Estrategia De Promoción De La Salud. Hacia la Promoción la Salud. 2011;16(1):202-18.

21. Guía de actividad física [Internet]. Organización Mundial de la Salud. 2016. Disponible en: http://whqlibdoc.who.int/publications/2010/978924 3599977_spa.pdf

22. Parisien M. Élaboration et mise a l'essai d'un programme communautaire d'exercices physiques visant á ámeliorer l'équilibre de seniors a risque de chutes. Rapport de travail dirigé au departement de médecine sociale et préventive. Université de Montréal. 1997.

23. Duncan P, Weiner D, Chandler J, Studenski S. Functional reach: A new clinical measure of balance. J Gerontol. 1990;45(6):192-7.

24. Cerda L. Manejo del trastorno de marcha del adulto mayor. Rev Médica Clínica Las Condes. 2014;25(2):265-75.

25. Silva Campos MP, Gomes Vianna LG, da Rocha Campos A. Os testes de equilíbrio Alcance Funcional e "Timed Up and Go" e o risco de quedas em idosos. Rev Kairós Gerontol. 2013;16(4):125-38.

26. Franchignoni F, Tesio L, Martino M, Ricupero C. Reliability of four simple, quantitative tests of balance and mobility in healthy elderly females. Aging Clin Exp Res. 1998;10(1):26-31.

27. Bohannon R. One-Legges balance test times. Vol. 78, Perceptual and Motor Skills. 1994. p. 801-2.

28. Schlicht J, Camaione D, Owen S. Effect of Intense Strength Training on Standing Balance, Walking Speed, and Sit-to-Stand Performance in Older Adults. Vol. 56, The Journals of Gerontology Series A: Biological Sciences and Medical Sciences. 2001. p. 281-6.

29. Csuka McCarty D. Simple method for measurement of lower extremity muscle strength. Am J Med. 1985;78(1):77-81.

30. Pereira FD, Batista WO, Furtado HL, Giani E de DAJTS, Dantas EHM. Comparação da força funcional de membros inferiores e superiores entre idosas fisicamente ativas e sedentárias. Rev Bras Geriatr Gerontol. 2009;12(3):417-27.

31. Ramírez-Villada JF, Chaparro-Obando D, León-Ariza $\mathrm{HH}$, Salazar Pachón J. Efecto del ejercicio físico para el control de los factores de riesgo cardiovascular modificables del adulto mayor: revisión sistemática. Rehabilitación. 2015 Sep;49(4):240-51.

32. Bhamani MA, Khan MM, Karim MS, Mir MU. Depression and its association with functional status and physical activity in the elderly in Karachi, Pakistan. Asian J Psychiatr. 2015 Apr;14:46-51.

33. Robitaille $Y$, Laforest S, Fournier M, Gauvin L, Parisien $\mathrm{M}$, Corriveau $\mathrm{H}$, et al. Moving Forward in Fall Prevention: An Intervention to Improve Balance Among Older Adults in Real-World Settings. Am J Public Health. 2005;95(11):2049-56.

34. Vera Sánchez M, Campillo Motilva R. Evaluación de la marcha y el equilibrio como factor de riesgo en las caídas del anciano. Rev Cuba Med Gen Integr [Internet]. 2003 [cited 2017 Mar 1];19(5). Disponible en: http://scielo.sld.cu/scielo.php?script=sci_arttext\&pid= S0864$21252003000500007 \& \operatorname{lng}=\mathrm{es} \& n r m=$ iso\&tlng=es

35. Correa JE, Gámez ER, Ibáñez M, Rodríguez KD. Aptitud física en mujeres adultas mayores vinculadas a un programa de envejecimiento activo. Rev Salud Uis. 
2011;43(3):263-70.

36. Filiatrault J, Parisien M, Laforest S, Genest C, Gauvin L, Fournier $M$, et al. Implantation $d$ ' un programme de prévention des chutes en milieu communautaire : du concept à la réalité *. 2008;1-21.

37. Fauchard T, Le Cren F. Présentation du programme intégré d'équilibre dynamique (PIED). Sci Sports. 2009;24(3):152-9.

38. Ledin T, Kronhed A, Möller C, Möller M. Effects of balance training in elderly evaluated by clinical tests and dynamic posturography. J Vestib Res Equilib Orientat. 1990;1(2):129-38. 